\title{
Editorial-Nutrition as supportive care in the cancer experience
}

\author{
Martin Chasen • Fredrick Ashbury
}

Received: 24 August 2009 / Accepted: 21 September 2009 /Published online: 6 October 2009

(C) Springer-Verlag 2009

Only a short while ago in the professional lives of most of our readers, the concept of "supportive care" for patients with cancer, their loved ones, and caregivers had very limited application. Oncology's goal was "cure" (sometimes at all costs), and as such, support for patients was focused on treating and potentially curing their cancers, rather than the more holistic approach of control and management of patients' demands. Working in parallel to this paradigm, and often in isolation, courageous individuals spearheaded innovative research, programs, and policies in supportive care in oncology. Their efforts have resulted in the more systematic identification and management of a wide range of issues, including quality of life, rehabilitation, communication, physical and mental health, late side effects of treatment, psychosocial needs, lifestyle factors, ethical dilemmas, and the challenges of survivorship across the life course (including pediatric cancers, youth, and young adults).

According to the World Health Organization, the burden of cancer has increased substantially to the point that cancer rates in the developing world are close to those of the socalled developed nations, due in part to changes in lifestyle (e.g., increased tobacco use), an increase in the average age

\footnotetext{
M. Chasen

Department of Oncology, McGill University,

Montreal, PQ, Canada

F. Ashbury

Division of Preventive Oncology, Department of Oncology, University of Calgary,

Calgary, AB, Canada

F. Ashbury $(\square)$

Dalla Lana School of Public Health, University of Toronto,

Toronto, ON, Canada

e-mail: fashbury@picepsconsultants.com
}

and size of populations, and improvements in the detection of cancer. Today, the number of persons having a direct experience with cancer is in the tens of millions, and we can expect increases in both incidence rates and prevalence that will require substantial financial and human resources. This "crisis of cancer" will demand our greater understanding of the supportive care issues of our patients, their loved ones, and caregivers.

Supportive Care in Cancer emerged nearly two decades ago to help "capture" and provide a vehicle for the dissemination of science-based, innovative approaches to this holistic oncology experience. These articles dedicated to nutrition issues in the cancer experience fit the journal's mandate perfectly. The articles promote appropriate nutrition for patients in preparation for, during and post therapy (up until very recently, most research on diet and nutrition has focused on causes and origins of disease and cancer prevention).

People diagnosed and living with cancer and their loved ones are profoundly interested in the role nutrition plays in the cancer experience (not just prevention, but how nutrition may enhance therapeutic benefits, ameliorate treatment toxicities, improve recovery patterns, reduce recurrence, and in cases of advanced cancer, improve the quality of end-of-life. Oncology professionals, therefore, have an obligation to research, discuss, and communicate the role of nutritional support in cancer care and the potential interaction of diet and nutrition with different therapies. The articles underscore the fact that decisions on nutrition (what foods, supplements, when to eat, how much, preparation) are informed when patients have access to professionals with different expertise. In addition, these articles emphasize that in order to build the evidence base needed to support informed decision making requires contributions from different disciplines working together. 
Input is necessary from patients, their loved ones, oncologists, dietitians and nutrition experts, epidemiologists, and persons versed in cross-cultural issues on nutrition.

The expanding role for nutrition gives us one more tool in the arsenal for supportive care in oncology. We are heartened by the increasing call and funding for nutrition research for survivors among national bodies, including government and not-for-profit agencies in countries around the world. We hope these articles stimulate further discussion and can do so only through their widespread dissemination to cancer professionals, scientists, policy makers, patients, and their loved ones. 